\title{
EDITORIAL
}

\section{COVID-19 AND OLDER ADULTS}

\author{
J.E. MORLEY ${ }^{1}$, B. VELLAS ${ }^{2}$ \\ 1. School of Social Work, Saint Louis University, St. Louis, Missouri, USA; 2. Gérontopôle, Department of Geriatrics, CHU Toulouse, Purpan University Hospital, Toulouse, France. \\ Corresponding author: John E. Morley, MB, BCh, Division of Geriatric Medicine, Saint Louis University School of Medicine, 1402 S. Grand Blvd., M238, St. Louis, MO 63104, \\ Email: john.morley@health.slu.edu
}

Key words: COVID-19, elderly, dyspnea.

"There is a significant probability of a large scale and lethal modern day pandemic occurring in our lifetimes." -Bill Gates

Since the great plague and cholera epidemics that occurred before the twentieth century, there have been a number of other pandemics starting with the Spanish Flu in 1918. In December, 2019, a new coronavirus, now recognized as COVID-19, began to cause respiratory illness in Wuhan, China. The epidemic began in a fish market and is most similar to snake, pangolin, horseshoe crab, and bat corona viruses. In humans it is spread by respiratory droplets. It can remain alive on plastic surfaces for over 72 hours. It is spread by respiratory droplets. At the Shattuck lecture in Boston in 2018. Bill Gates called for a "clear road map for a comprehensive pandemic preparedness and response system (1)."

COVID-19 presents with nasal secretions, cough, dyspnea, fever, myalgia and occasionally diarrhea. Around $15 \%$ may go on to develop acute respiratory distress syndrome for 5 days, but may last up to 14 days. Viral shedding may last up to 37 days. Over $95 \%$ of hospitalized patients have abnormal chest computed tomography (2). On CT, ground glass opacities with a reticular pattern, a subplural line, fibrotic streaks and an air bronchogram were the most common signs (3). These findings allowed COVID-19 pneumonia to be separated from classical viral pneumonia. From the laboratory point of view lymphocytopenia, elevated C-reactive protein, elevated interleukin-6, elevated lactic dehydrogenase, hypoalbuminemia, a decreased CD8 count increased ferritin and decreased procalcitonin (4). In addition, very high angiotensin II levels were present. Highly elevated d-dimer levels are associated with mortality for people on ventilators.

Besides acute respiratory distress syndrome severely ill patients develop myocardial damage and this is associated with increased mortality. Kidney and liver disease also occur. COVID-19 enters the central nervous system and increases inflammatory cytokines which can be expected to lead to delirium. Older people also have an increase in delirium and do not always have an increase in fever.

The prevalence of COVID-19 in the community is uncertain as it appears a number of persons may not show symptoms.
It would appear that the mortality may be as low as $0.6 \%$ (5). It is clear that older persons are at a much higher risk of mortality (about 15\%) than younger persons (5). Persons with comorbidity are at an increased risk. It is suggested that the FRAIL screen is used to detect persons at increased risk (6, $7,8)$. Persons with hypertension and diabetes mellitus are at increased risk possibly due to alterations in the angiotensin converting enzyme 2 (ACE 2) receptor produced by ACE 1 inhibitors.

Primary prevention especially for older persons with comorbidity is social distancing and where possible social isolation. For older persons the problem with social isolation is loneliness (9). Loneliness leads to depression, cognitive dysfunction, disability, cardiovascular disease and increased mortality. Obviously, prevention also requires regular hand washing and cleaning of surfaces. Wearing a mask does not provide protection for the individual. Finally, the first vaccine has just started testing. If it or other vaccines under development mount an adequate antibody response there will be a need to try to rapidly bring it to the general public. It is important to recognize that some persons, like "Typhoid Mary" who spread typhoid fever in the 1910s, may be asymptomatic. Thus, distance must be kept from everybody.

At present, while there are no established drugs to treat COVID-19, some are showing promise. Chloroquine phosphate, an anti-malarial, has been shown to be useful in treating COVID-19 pneumonia (10). Remdesivir, an antiviral drug developed to treat Ebola, has been suggested to have positive effects in COVID-19 infected patients with severe respiratory disease (11). These patients developed gastrointestinal symptoms and elevated liver function tests. Some patients with severe COVID-19 disease develop cytokine storm and this may be prevented with toclizumab. Passive infusion of polyclonal plasma antibodies from persons who have had COVID-19 infection has been suggested and monoclonal antibodies to COVID-19 are under development (12). Finally, COVID-19 binds to the soluble portion of the ACE-2 receptor and this seems essential for the virus to enter cells. The possibility of a monoclonal antibody to the soluble portion of the ACE-2 receptor is under consideration. It is uncertain whether stopping the use of ACE 1 inhibitors which increase ACE- 2 receptors should be undertaken. However, this 
may explain the increased virulence of COVID-19 in persons with hypertension and diabetes mellitus. COVID-19 infected patients may do worse if taking ibuprofen, so it is recommended that patients take acetaminophen or paracetamol for fever and pain.

In conclusion, COVID-19 represents a major threat to older adults. This is particularly true in older persons with frailty and co-morbidity. Other factors that appear to play a role in the increased severity in older persons are the decline in immune function and alterations in the ACE 2 receptor. There is need for rapid development of a COVID-19 vaccine and its deployment among the population. In the meantime, social distancing, careful hand washing and using antiseptic wipes to clean surfaces and door handles before touching them represent the appropriate preventive measures. During the pandemic it is especially important to isolate older persons in nursing homes and to provide support when nursing home staff need to be quarantined. With good population health approaches, it is expected that the COVID-19 pandemic will be controlled in a relatively short time period.

Acknowledgements: This article was developed by the Geriatric Work Force Enhancement Program funded by HRSA.

Disclosures: The authors declare there are no conflicts.

\section{References}

1. Gates B. Innovation for pandemics. N Engl J Med 2018;378:2057-2060.

2. Sun P, Qie S, Liu Z, et al. Clinical characteristics of 50,466 hospitalized patients with 2019-nCoV infection. J Med Virol 2020;Feb 28. Doi: 10.1002/jmv.25735.

3. Wang YXJ, Liu WH, Yang M, Chen W. The role of CT for Covid-19 patient's management remains poorly defined. Ann Transl Med 2020;8:145.

4. Liu Y, Yang Y, Zhang C, et al. Clinical and biochemical indexes from 2019 $\mathrm{nCoV}$ infected patients linked to viral loads and lung injury. Sci China Life Sci 2020;63:364-374.

5. Wilson N, Kvalsvig A, Barnard LT, Baker MG. Case-fatality risk estimates for COVID-19 calculated by using a lag time for fatality. Emerg Infect Ids 2020;26(6) doi: 10.3201/eid2606.200320 [Epub ahead of print].

6. Dent E, Morley JE, Cruz-Jentoft AJ, et al. Physical frailty: ICFSR International Clinical Practice Guidelines for identification and management. J Nutr Health Aging 2019;23(9):771-787.

7. Aprahamian I, Arico de Almeida GV, de Vascocellos Romanin CF, et al. Frailty could predict death in older adults after admission $t$ emergency department? A 6-month prospective study from a middle-income country. J Nutr Health Aging 2019;23:641-647.

8. Woo J, Yang X, Tin Lui L, Li Q, et al. Utility of the FRAIL questionnaire in detecting heart failure with preserved ejection fraction. J Nutr Health Aging 2019;23:373-377.

9. Berg-Weger M, Morley J. Editorial: Loneliness in old age: An unaddressed health problem. J Nutr Health Aging 2020;24:243-245.

10. Colson P, Rolain JM, Lagier JC, et al. Chloroquine and hydroxychloroquine as available weapons to fight COVID-19. Int J Antimicrob Agents 2020; Mar 4: 105932 doi: 10.1016/j.ijantimicag.2020.105932. [Epub ahead of print].

11. Al-Tawfiq JA, Al-Homoud AH, Memish ZA. Remdesivir as a possible therapeutic option for the COVID-19. Travel Med Infect Dis 2020; Mar 5:101615. Doi 10.1016/j.tmaid.2020.101615.

12. Martinez MA. Compounds with therapeutic potential against novel respiratory 2019 coronavirus. Antimicrob Agents Chemother 2020 Mar 9. Pii: AAC.00399-20. Doi: 10.1128/AAC.00399-20. [Epub ahead of print]. 